\title{
Order estimates of the uniform approximations by Zygmund sums on the classes of convolutions of periodic functions
}

\section{Serdyuk A.S. ${ }^{1}$, Hrabova U.Z. ${ }^{2}$}

The Zygmund sums of a function $f \in L_{1}$ are trigonometric polynomials of the form $Z_{n-1}^{s}(f ; t):=$ $\frac{a_{0}}{2}+\sum_{k=1}^{n-1}\left(1-\left(\frac{k}{n}\right)^{s}\right)\left(a_{k}(f) \cos k t+b_{k}(f) \sin k t\right), s>0$, where $a_{k}(f)$ and $b_{k}(f)$ are the Fourier coefficients of $f$. We establish the exact-order estimates of uniform approximations by the Zygmund sums $Z_{n-1}^{s}$ of $2 \pi$-periodic continuous functions from the classes $C_{\beta, p}^{\psi}$. These classes are defined by the convolutions of functions from the unit ball in the space $L_{p}, 1 \leq p<\infty$, with generating fixed kernels $\Psi_{\beta}(t) \sim \sum_{k=1}^{\infty} \psi(k) \cos \left(k t+\frac{\beta \pi}{2}\right), \Psi_{\beta} \in L_{p^{\prime}}, \beta \in \mathbb{R}, \frac{1}{p}+\frac{1}{p^{\prime}}=1$. We additionally assume that the product $\psi(k) k^{s+1 / p}$ is generally monotonically increasing with the rate of some power function, and, besides, for $1<p<\infty$ it holds that $\sum_{k=n}^{\infty} \psi^{p^{\prime}}(k) k^{p^{\prime}-2}<\infty$, and for $p=1$ the following condition $\sum_{k=n}^{\infty} \psi(k)<\infty$ is true.

It is shown, that under these conditions Zygmund sums $Z_{n-1}^{s}$ and Fejér sums $\sigma_{n-1}=Z_{n-1}^{1}$ realize the order of the best uniform approximations by trigonometric polynomials of these classes, namely for $1<p<\infty$

$$
E_{n}\left(C_{\beta, p}^{\psi}\right)_{C} \asymp \mathcal{E}\left(C_{\beta, p}^{\psi} ; Z_{n-1}^{s}\right)_{C} \asymp\left(\sum_{k=n}^{\infty} \psi^{p^{\prime}}(k) k^{p^{\prime}-2}\right)^{1 / p^{\prime}}, \quad \frac{1}{p}+\frac{1}{p^{\prime}}=1 ，
$$

and for $p=1$

$$
E_{n}\left(C_{\beta, 1}^{\psi}\right)_{C} \asymp \mathcal{E}\left(C_{\beta, 1}^{\psi} ; Z_{n-1}^{s}\right)_{C} \asymp \begin{cases}\sum_{k=n}^{\infty} \psi(k), & \cos \frac{\beta \pi}{2} \neq 0, \\ \psi(n) n, & \cos \frac{\beta \pi}{2}=0\end{cases}
$$

where

$$
E_{n}\left(C_{\beta, p}^{\psi}\right)_{C}:=\sup _{f \in C_{\beta, p}^{\psi}} \inf _{t_{n-1} \in \mathcal{T}_{2 n-1}}\left\|f(\cdot)-t_{n-1}(\cdot)\right\|_{C},
$$

and $\mathcal{T}_{2 n-1}$ is the subspace of trigonometric polynomials $t_{n-1}$ of order $n-1$ with real coefficients,

$$
\mathcal{E}\left(C_{\beta, p}^{\psi} ; Z_{n-1}^{s}\right)_{C}:=\sup _{f \in C_{\beta, p}^{\psi}}\left\|f(\cdot)-Z_{n-1}^{s}(f ; \cdot)\right\|_{C} .
$$

Key words and phrases: best approximation, Zygmund sum, Fejér sum, subspace of trigonometric polynomials, order estimate.

${ }^{1}$ Institute of Mathematics, National Academy of Sciences of Ukraine, 3 Tereschenkivska str., 01601, Kyiv, Ukraine

2 Lesya Ukrainka Volyn National University, 9 Potapova str., 43025, Lutsk, Ukraine

E-mail: serdyuk@imath.kiev.ua (Serdyuk A.S.), grabova_u@ukr.net (Hrabova U.Z.) 


\section{Notations, definitions and auxiliary statements}

Denote by $L_{p}, 1 \leq p \leq \infty$, the space of $2 \pi$-periodic summable on $[0,2 \pi]$ functions $f$ with the norm

$$
\|f\|_{p}= \begin{cases}\left(\int_{0}^{2 \pi}|f(t)|^{p} d t\right)^{1 / p}, & 1 \leq p<\infty, \\ \underset{t}{\operatorname{ess} \sup }|f(t)|, & p=\infty\end{cases}
$$

and by $C$ the space of $2 \pi$-periodic continuous functions with the norm defined by the equality $\|f\|_{C}=\max _{t}|f(t)|$.

Let $f \in L_{1}$ and

$$
S[f](x)=\frac{a_{0}}{2}+\sum_{k=1}^{\infty}\left(a_{k}(f) \cos k x+b_{k}(f) \sin k x\right),
$$

be the Fourier series of the function $f$.

If for a sequence $\psi(k) \in \mathbb{R}$ and fixed number $\beta \in \mathbb{R}$ the series

$$
\sum_{k=1}^{\infty} \frac{1}{\psi(k)}\left(a_{k}(f) \cos \left(k x+\frac{\beta \pi}{2}\right)+b_{k}(f) \sin \left(k x+\frac{\beta \pi}{2}\right)\right)
$$

is the Fourier series of a summable function $\varphi$, then this function is called a $(\psi, \beta)$-derivative of the function $f$ and is denoted by $f_{\beta}^{\psi}$. A set of functions, for which this condition is satisfied, is denoted by $L_{\beta}^{\psi}$, and subset all continuous functions from $L_{\beta}^{\psi}$ is denoted by $C_{\beta}^{\psi}$.

If $f \in L_{\beta}^{\psi}$ and furthermore $f_{\beta}^{\psi} \in \mathfrak{N}$, where $\mathfrak{N} \subset L_{1}$, then we write that $f \in L_{\beta}^{\psi} \mathfrak{N}$. Let us put $L_{\beta}^{\psi} \mathfrak{N} \cap C=C_{\beta}^{\psi} \mathfrak{N}$. The concept of $(\psi, \beta)$-derivative is a natural generalization of the concept of $(r, \beta)$-derivative in the Weyl-Nagy sense and coincides almost everywhere with the last one, when $\psi(k)=k^{-r}, r>0$. Namely, in this case $L_{\beta}^{\psi} \mathfrak{N}=W_{\beta}^{r} \mathfrak{N}, f_{\beta}^{\psi}=f_{\beta}^{r}$, where $f_{\beta}^{r}$ is the derivative in the Weyl-Nagy sense, and $W_{\beta}^{r} \mathfrak{N}$ are the Weyl-Nagy classes [22], [20]. In the case $\beta=r$, the classes $W_{\beta}^{r} \mathfrak{N}$ are the well known Weyl classes $W_{r}^{r} \mathfrak{N}$, while the derivatives $f_{\beta}^{r}$ coincide almost everywhere with the derivatives in the sense of Weyl $f_{r}^{r}$. If, in addition, $\beta=r, r \in \mathbb{N}$, then $f_{\beta}^{r}$ coincide almost everywhere with the usual derivatives $f^{(r)}$ of the order $r$ of the function $f$ $\left(f_{\beta}^{r}=f_{r}^{r}=f^{(r)}\right)$ and at the same time $W_{\beta}^{r} \mathfrak{N}=W_{r}^{r} \mathfrak{N}=W^{r} \mathfrak{N}$.

According to [20, Statement 3.8.3], if the series

$$
\sum_{k=1}^{\infty} \psi(k) \cos \left(k t-\frac{\beta \pi}{2}\right), \quad \beta \in \mathbb{R}
$$

is the Fourier series of the function $\Psi_{\beta} \in L_{1}$, then the elements $f$ of the classes $L_{\beta}^{\psi} \mathfrak{N}$ for almost every $x \in \mathbb{R}$ are represented as the convolution

$$
f(x)=\frac{a_{0}}{2}+\left(\Psi_{\beta} * \varphi\right)(x)=\frac{a_{0}}{2}+\frac{1}{\pi} \int_{-\pi}^{\pi} \Psi_{\beta}(x-t) \varphi(t) d t, \quad a_{0} \in \mathbb{R}, \varphi \perp 1, \varphi \in \mathfrak{N},
$$

where $\varphi$ almost everywhere coincides with $f_{\beta}^{\psi}$.

As sets $\mathfrak{N}$ we will consider the unit balls of the spaces $L_{p}$ :

$$
U_{p}=\left\{\varphi \in L_{p}:\|\varphi\|_{p} \leq 1\right\}, \quad 1 \leq p \leq \infty .
$$


Then put: $L_{\beta, p}^{\psi}:=L_{\beta}^{\psi} U_{p}, C_{\beta, p}^{\psi}:=C_{\beta}^{\psi} U_{p}, W_{\beta, p}^{r}:=W_{\beta}^{r} U_{p}$.

According to [20, Statement 1.2], if the fixed kernel $\Psi_{\beta}$ of the classes $L_{\beta, p}^{\psi}$ and $C_{\beta, p}^{\psi}$ satisfies the inclusion $\Psi_{\beta} \in L_{p^{\prime}}, \frac{1}{p}+\frac{1}{p^{\prime}}=1,1 \leq p \leq \infty$, then the convolutions of the form (1) are continuous functions, where $\mathfrak{N}=U_{p}$. It is clear that in this case for $f \in C_{\beta, p}^{\psi}$ the equality (1) is fulfilled for all $x \in \mathbb{R}$.

We assume that the sequences $\psi(k)$ are traces on the set of natural numbers $\mathbb{N}$ of some positive continuous convex downwards functions $\psi(t)$ of the continuous argument $t \geq 1$, that tends to zero for $t \rightarrow \infty$. The set of all such functions $\psi(t)$ is denoted by $\mathfrak{M}$.

To classify functions $\psi$ from $\mathfrak{M}$ on their speed of decreasing to zero it is convenient to use the following characteristic

$$
\alpha(t)=\alpha(\psi ; t)=\frac{\psi(t)}{t\left|\psi^{\prime}(t)\right|^{\prime}}, \quad \psi^{\prime}(t):=\psi^{\prime}(t+0) .
$$

With its help we consider the following subsets of the set $\mathfrak{M}$ (see, e.g. [20])

$$
\begin{gathered}
\mathfrak{M}_{0}:=\{\psi \in \mathfrak{M}: \exists K>0 \forall t \geq 10<K \leq \alpha(\psi ; t)\}, \\
\mathfrak{M}_{C}:=\left\{\psi \in \mathfrak{M}: \quad \exists K_{1}, K_{2}>0 \forall t \geq 10<K_{1} \leq \alpha(\psi ; t) \leq K_{2}\right\} .
\end{gathered}
$$

It is clear that $\mathfrak{M}_{C} \subset \mathfrak{M}_{0}$.

Zygmund sums of the order $n-1$ of the function $f \in L_{1}$ are the trigonometric polynomials of the form

$$
Z_{n-1}^{s}(f ; t)=\frac{a_{0}}{2}+\sum_{k=1}^{n-1}\left(1-\left(\frac{k}{n}\right)^{s}\right)\left(a_{k}(f) \cos k t+b_{k}(f) \sin k t\right), \quad s>0,
$$

where $a_{k}(f)$ and $b_{k}(f)$ are Fourier coefficients of the function $f$.

In the case $s=1$ polynomials $Z_{n-1}^{s}$ are Fejér sums

$$
Z_{n-1}^{1}(f ; t)=: \sigma_{n-1}(f ; t)=\frac{a_{0}}{2}+\sum_{k=1}^{n-1}\left(1-\frac{k}{n}\right)\left(a_{k}(f) \cos k t+b_{k}(f) \sin k t\right) .
$$

In this paper we consider the following approximation characteristics

$$
\mathcal{E}\left(C_{\beta, p}^{\psi} ; Z_{n-1}^{s}\right)_{C}=\sup _{f \in C_{\beta, p}^{\psi}}\left\|f(\cdot)-Z_{n-1}^{s}(f ; \cdot)\right\|_{C}, \quad 1 \leq p \leq \infty, \quad \beta \in \mathbb{R},
$$

and solve the problem of establishing the order of decreasing to zero as $n \rightarrow \infty$ of the mentioned quantities with respect to relations between parameters $\psi, \beta, p$ and $s$. It is clear that we can make conclusion about the approximation ability of a linear polynomial approximation method (including Fejér $\sigma_{n-1}$ and Zygmund $Z_{n-1}^{s}$ methods) on the class $C_{\beta, p^{\prime}}^{\psi}$ after comparison the rate of decreasing of the exact upper bounds of uniform deviations of trigonometric sums, which are generated by this method, on the set $C_{\beta, p}^{\psi}$ with the rate of decreasing of the best uniform approximations of the class $C_{\beta, p}^{\psi}$ by trigonometric polynomials $t_{n-1}$ of order not higher than $n-1$, namely the quantities of the form

$$
E_{n}\left(C_{\beta, p}^{\psi}\right)_{C}=\sup _{f \in C_{\beta, p}^{\psi}} \inf _{t_{n-1}}\left\|f(\cdot)-t_{n-1}(\cdot)\right\|_{C}, \quad 1 \leq p \leq \infty,
$$


where $\mathcal{T}_{2 n-1}$ is the subspace of trigonometric polynomials $t_{n-1}$ of order $n-1$ with real coefficients. In this case, since always the following estimate holds

$$
E_{n}\left(C_{\beta, p}^{\psi}\right)_{C} \leq \mathcal{E}\left(C_{\beta, p}^{\psi} ; Z_{n-1}^{s}\right)_{C^{\prime}} \quad n \in \mathbb{N},
$$

it is important to know under which restrictions on the parameters $\psi, s, \beta$ and $p$ the following equality takes place

$$
E_{n}\left(C_{\beta, p}^{\psi}\right)_{C} \asymp \mathcal{E}\left(C_{\beta, p}^{\psi} ; Z_{n-1}^{s}\right)_{C} \cdot
$$

The notation $A(n) \asymp B(n)$ means, that $A(n)=O(B(n))$ and at the same time $B(n)=O(A(n))$, where by the notation $A(n)=O(B(n))$ we mean, that there exists a constant $K>0$ such that the inequality $A(n) \leq K(B(n))$ holds.

In the work [27] A. Zygmund introduced trigonometric polynomials of the form (2) and found exact order estimates of the quantities $\mathcal{E}\left(W_{\infty}^{r} ; Z_{n-1}^{s}\right)_{C}$ at $r \in \mathbb{N}$. B. Nagy investigated in [7] the quantities $\mathcal{E}\left(W_{\beta, \infty}^{r} ; Z_{n-1}^{s}\right)_{C}$ at $r>0, \beta \in \mathbb{Z}$, and for $s \leq r$ he established the asymptotic equality, and for $s>r$ he found order estimates. Later, S.A. Telyakovsky [23] obtained asymptotically exact equalities for the quantities $\mathcal{E}\left(W_{\beta, \infty}^{r} ; Z_{n-1}^{s}\right)_{C}$ for $r>0$ and $\beta \in \mathbb{R}$ for $n \rightarrow \infty$. On the Weyl-Nagy classes, the exact order estimates of the quantities $\mathcal{E}\left(W_{\beta, p}^{r} ; Z_{n-1}^{s}\right)_{C}$ for $1<p<\infty$ and $r>1 / p$ and for $p=1$ and $r \geq 1, \beta \in \mathbb{R}$ are found in the work [6].

Concerning the Fejér sums $\sigma_{n-1}(f ; t)$ it should be noticed that the order estimates of quantities $\mathcal{E}\left(W_{\beta, \infty}^{r} ; \sigma_{n-1}\right)_{\mathcal{C}^{\prime}} r>0$, for $\beta \in \mathbb{Z}$ were found by S.M. Nikol'skii [8]; for the quantities $\mathcal{E}\left(W_{r, p}^{r} ; \sigma_{n-1}\right)_{C}$ for $1<p \leq \infty$ and $r>1 / p$, and also for $p=1$ and $r \geq 1$ were found by V.M. Tikhomirov [25] and by A.I. Kamzolov [5].

Approximation properties of Zygmund sums on the classes of $(\psi, \beta)$-differentiable functions were studied in the works [2,14,15], (see also [20]). Particularly in the work [2] of D.M. Bushev the asymptotic equalities for the quantities $\mathcal{E}\left(C_{\beta, \infty}^{\psi} ; Z_{n-1}^{s}\right)_{C}$ were established for some quite natural constraints on $\psi$ and $s$ as $n \rightarrow \infty$. In the case, when the series $\sum_{k=1}^{\infty} \psi^{2}(k)$ is convergent, the exact values of the quantities $\mathcal{E}\left(C_{\beta, 2}^{\psi} ; Z_{n-1}^{s}\right)$ were established in the work [15] of A.S. Serdyuk and I.V.Sokolenko.

In the work [14], the authors found the exact order estimatites of uniform approximations by Zygmund sums $Z_{n-1}^{s}$ on the classes $C_{\beta, p^{\prime}}^{\psi}, 1<p<\infty$, when $\psi \in \Theta_{p}$, and $\Theta_{p}, 1<p<\infty$, is the set of non-increasing functions $\psi(t)$, for which there exists $\alpha>1 / p$ such that the function $t^{\alpha} \psi(t)$ almost decreases, and $\psi(t) t^{s+1 / p-\varepsilon}$ increases on $[1, \infty)$ for some $\varepsilon>0$.

Concerning the estimates of the best uniform approximations of functional compacts, it should be noticed the following. For the Weyl-Nagy classes $W_{\beta, p}^{r}, r>1 / p, \beta \in \mathbb{R}, 1 \leq p \leq \infty$, the exact order estimates of the best approximations $E_{n}\left(W_{\beta, p}^{r}\right)_{C}$ are known (see, e.g. [24]). Moreover, for $p=\infty$ the exact values of the quantities $E_{n}\left(W_{\beta, \infty}^{r}\right)_{C}$ for all $r>0, \beta \in \mathbb{R}$ and $n \in \mathbb{N}$ are known (see [3]).

The order estimates of the best approximations of the classes $C_{\beta, p}^{\psi}$ under certain restrictions on $\psi, \beta$ and $p$ were investigated in the works $[4,17,18,20]$. In some partial cases (especially for $p=\infty)$ the exact or asymptotically exact values of the quantities $E_{n}\left(C_{\beta, p}^{\psi}\right)_{C}$ are also known (see $[9-13,16,20])$. 
In this paper, we establish the exact order estimates of the quantities of the form (3) for all $1 \leq p<\infty$ and $\beta \in \mathbb{R}$, in case, when $\psi(t) t^{1 / p} \in \mathfrak{M}_{0}$, the product $\psi(k) k^{s+1 / p}$ generally monotonically increases, $\psi(k) k^{s+1 / p-\varepsilon}$ almost increases (according to Bernstein) for some $\varepsilon>0$ and for $1<p<\infty$

$$
\sum_{k=n}^{\infty} \psi^{p^{\prime}}(k) k^{p^{\prime}-2}<\infty, \quad \frac{1}{p}+\frac{1}{p^{\prime}}=1
$$

and for $p=1$

$$
\sum_{k=n}^{\infty} \psi(k)<\infty
$$

The conditions (6) and (7) and the monotonic decreasing to zero of the sequence $\psi(k)$ ensure the inclusion $\Psi_{\beta} \in L_{p^{\prime}}, 1 / p+1 / p^{\prime}=1,1 \leq p<\infty$ (see, e.g. [28, Lemma 12.6.6, p. 193]).

In this paper, it is also shown that for some conditions Zygmund sums (and at $s=1$ also the Fejér sums) realize the orders of the best uniform approximations on the classes $C_{\beta, p^{\prime}}^{\psi}$, that is the order estimate (5) is true. Previously, this property was proved for Fourier sums $[4,18,19,21]$.

Let us formulate some necessary definitions.

A non-negative sequence $a=\left\{a_{k}\right\}_{k=1}^{\infty}, k \in \mathbb{N}$, is said to be generally monotonically increasing (we write $a \in G M^{+}$), if there exists a constant $A \geq 1$, such that for any natural $n_{1}$ and $n_{2}$ such that $n_{1} \leq n_{2}$ the inequalities

$$
a_{n_{1}}+\sum_{k=n_{1}}^{m-1}\left|a_{k}-a_{k+1}\right| \leq A a_{m}, \quad m=\overline{n_{1}, n_{2}}
$$

hold (see, e.g. [1, p. 811]). It is easy to see that if the positive sequence $a=\left\{a_{k}\right\}_{k=1}^{\infty}$ increases, starting from some number, then it generally monotonically increasing.

A non-negative sequence $a=\left\{a_{k}\right\}_{k=1}^{\infty}, k \in \mathbb{N}$, is said to be almost increasing (according to Bernstein, see, e.g. [26, p. 730]) if there exists a constant $K$, such that for all $n_{1} \leq n_{2}$ we have

$$
a_{n_{1}} \leq K a_{n_{2}}
$$

In this case, if for the sequence $a=\left\{a_{k}\right\}_{k=1}^{\infty}$ there exists a constant $\varepsilon>0$, such that $\left\{a_{k} k^{-\varepsilon}\right\}$ almost increases, then we write $a \in G A^{+}$. It is clear that if the sequence $a$ belongs to $G M^{+}$, then it is almost increasing according to Bernstein.

Let us put further $g_{\delta}(t):=\psi(t) t^{\delta}, t \in[1, \infty)$ with $\delta>0$.

\section{Order estimates of the approximations by Zygmund sums on the classes of convolutions}

Theorem 1. Let $s>0,1 \leq p<\infty, g_{1 / p} \in \mathfrak{M}_{0,}, g_{s+1 / p} \in G M^{+} \cap G A^{+}, \beta \in \mathbb{R}$ and $n \in \mathbb{N}$. In the case $1<p<\infty$, if the condition (6) holds and the inequality

$$
\inf _{t \geq 1} \alpha\left(g_{1 / p} ; t\right)>\frac{p^{\prime}}{2}
$$

holds, then the following order estimates take place

$$
E_{n}\left(C_{\beta, p}^{\psi}\right)_{C} \asymp \mathcal{E}\left(C_{\beta, p}^{\psi} ; Z_{n-1}^{s}\right)_{C} \asymp\left(\sum_{k=n}^{\infty} \psi^{p^{\prime}}(k) k^{p^{\prime}-2}\right)^{1 / p^{\prime}}, \quad \frac{1}{p}+\frac{1}{p^{\prime}}=1 ;
$$


in the case $p=1$, if the condition (7) holds and the inequality

$$
\inf _{t \geq 1} \alpha\left(g_{1} ; t\right)>1
$$

holds, then the following order estimates take place

$$
E_{n}\left(C_{\beta, 1}^{\psi}\right)_{C} \asymp \mathcal{E}\left(C_{\beta, 1}^{\psi} ; Z_{n-1}^{s}\right)_{C} \asymp \begin{cases}\sum_{k=n}^{\infty} \psi(k), & \cos \frac{\beta \pi}{2} \neq 0, \\ \psi(n) n, & \cos \frac{\beta \pi}{2}=0 .\end{cases}
$$

Proof. Since the operator $Z_{n-1}^{s}: f(t) \rightarrow Z_{n-1}^{s}(f, t)$ is linear polynomial operator, which is invariant under the shift, i.e.

$$
Z_{n-1}^{s}\left(f_{h}, t\right)=Z_{n-1}^{s}(f, t+h), \quad f_{h}(t)=f(t+h), \quad h \in \mathbb{R},
$$

and norm in $C$ and classes $C_{\beta, p}^{\psi}$ also are invariant under the shift, that is

$$
\left\|f_{h}\right\|_{C}=\|f\|_{C} ; \quad f(t) \in C_{\beta, p}^{\psi} \Rightarrow f_{h}(t) \in C_{\beta, p^{\prime}}^{\psi}
$$

then

$$
\mathcal{E}\left(C_{\beta, p}^{\psi} ; Z_{n-1}^{s}\right)_{C}=\sup _{f \in C_{\beta, p}^{\psi}}\left|f(0)-Z_{n-1}^{s}(f ; 0)\right| .
$$

By virtue of (1) and (2) for any function $f \in C_{\beta, p^{\prime}}^{\psi} 1 \leq p<\infty, \beta \in \mathbb{R}, s>0$, the following equality holds

$$
f(0)-Z_{n-1}^{s}(f ; 0)=\frac{1}{\pi} \int_{-\pi}^{\pi}\left(\frac{1}{n^{s}} \sum_{k=1}^{n-1} \psi(k) k^{s} \cos \left(k t+\frac{\beta \pi}{2}\right)+\Psi_{-\beta, n}(t)\right) \varphi(t) d t,
$$

where $\Psi_{-\beta, n}(t)=\sum_{k=n}^{\infty} \psi(k) \cos \left(k t+\frac{\beta \pi}{2}\right),\|\varphi\|_{p} \leq 1, n \in \mathbb{N}$.

Relations (14) and (15), Hölder's inequality and triangle inequality imply that for $1 \leq p<\infty$

$$
\begin{aligned}
\mathcal{E}\left(C_{\beta, p^{\prime}}^{\psi} ; Z_{n-1}^{s}\right)_{C} & \leq \frac{1}{\pi}\left\|\frac{1}{n^{s}} \sum_{k=1}^{n-1} \psi(k) k^{s} \cos \left(k t+\frac{\beta \pi}{2}\right)+\Psi_{-\beta, n}(t)\right\|_{p^{\prime}} \\
& \leq \frac{1}{\pi n^{s}}\left\|\sum_{k=1}^{n-1} \psi(k) k^{s} \cos \left(k t+\frac{\beta \pi}{2}\right)\right\|_{p^{\prime}}+\frac{1}{\pi}\left\|\Psi_{-\beta, n}(t)\right\|_{p^{\prime}}, \frac{1}{p}+\frac{1}{p^{\prime}}=1 .
\end{aligned}
$$

Let us show that, if $g_{s+1 / p} \in G M^{+} \cap G A^{+}$, where $g_{s+1 / p}=\left\{\psi(k) k^{s+1 / p}\right\}_{k=1}^{\infty}$, then

$$
\left\|\sum_{k=1}^{n-1} \psi(k) k^{s} \cos \left(k t+\frac{\beta \pi}{2}\right)\right\|_{p^{\prime}}=O\left(\psi(n) n^{s+\frac{1}{p}}\right), \quad 1 \leq p<\infty .
$$

Applying Abel transformation to the function $\sum_{k=1}^{n-1} \psi(k) k^{s} \cos \left(k t+\frac{\beta \pi}{2}\right)$, we have

$$
\begin{aligned}
\sum_{k=1}^{n-1} \psi(k) k^{s} \cos \left(k t+\frac{\beta \pi}{2}\right)=\sum_{k=1}^{n-2} & \left(\psi(k) k^{s}-\psi(k+1)(k+1)^{s}\right) D_{k, \beta}(t) \\
& +\psi(n-1)(n-1)^{s} D_{n-1, \beta}(t)-\frac{1}{2} \cos \frac{\beta \pi}{2}
\end{aligned}
$$


where

$$
D_{k, \beta}(t):=\frac{1}{2} \cos \frac{\beta \pi}{2}+\sum_{v=1}^{k} \cos \left(v t-\frac{\beta \pi}{2}\right) .
$$

Then, in view of $\left\|D_{k, \beta}(\cdot)\right\|_{p^{\prime}}=O\left(k^{1-\frac{1}{p^{\prime}}}\right)=O\left(k^{\frac{1}{p}}\right), 1 \leq p<\infty, k \in \mathbb{N}, \beta \in \mathbb{R}$, (see, e.g. [4]) from (18) we get

$$
\begin{aligned}
\left\|\sum_{k=1}^{n-1} \psi(k) k^{s} \cos \left(k t+\frac{\beta \pi}{2}\right)\right\|_{p^{\prime}}=O(1) & +O\left(\sum_{k=1}^{n-2}\left|\psi(k) k^{s}-\psi(k+1)(k+1)^{s}\right| k^{\frac{1}{p}}\right) \\
& +O\left(\psi(n-1)(n-1)^{s+\frac{1}{p}}\right) .
\end{aligned}
$$

Since $g_{s+1 / p} \in G M^{+}$, then, by using the triangle inequality, inequality (8) and Lagrange theorem, we have

$$
\begin{aligned}
\sum_{k=1}^{n-2}\left|\psi(k) k^{s}-\psi(k+1)(k+1)^{s}\right| k^{\frac{1}{p}} \leq & \sum_{k=1}^{n-2}\left|\psi(k) k^{s+\frac{1}{p}}-\psi(k+1)(k+1)^{s+\frac{1}{p}}\right| \\
& +\sum_{k=1}^{n-2}\left|\psi(k+1)(k+1)^{s+\frac{1}{p}}-\psi(k+1)(k+1)^{s} k^{\frac{1}{p}}\right| \\
\leq & A \psi(n-1)(n-1)^{s+\frac{1}{p}}+\frac{1}{p} \sum_{k=1}^{n-2} \psi(k+1)(k+1)^{s} k^{\frac{1}{p}-1} \\
\leq & A \psi(n-1)(n-1)^{s+\frac{1}{p}}+2 \sum_{k=2}^{n-1} \frac{\psi(k) k^{s+\frac{1}{p}}}{k} .
\end{aligned}
$$

According to the condition $g_{s+1 / p} \in G A^{+}$, there exits $\varepsilon>0$ such that the sequence $\left\{g_{s+1 / p}(k) k^{-\varepsilon}\right\}=\left\{\psi(k) k^{s+1 / p-\varepsilon}\right\}$ almost increases, and hence taking into account (9), we obtain

$$
\begin{aligned}
\sum_{k=2}^{n-1} \frac{\psi(k) k^{s+1 / p}}{k} & =\sum_{k=2}^{n-1} \frac{\psi(k) k^{s+1 / p-\varepsilon}}{k^{1-\varepsilon}} \leq K \psi(n-1)(n-1)^{s+1 / p-\varepsilon} \sum_{k=2}^{n-1} \frac{1}{k^{1-\varepsilon}} \\
& <K \psi(n-1)(n-1)^{s+1 / p-\varepsilon} \int_{1}^{n-1} \frac{d t}{t^{1-\varepsilon}}<\frac{K}{\varepsilon} \psi(n-1)(n-1)^{s+1 / p}
\end{aligned}
$$

From (20) and (21) we get the following inequality

$$
\left|\psi(k) k^{s}-\psi(k+1)(k+1)^{s}\right| k^{\frac{1}{p}} \leq\left(A+\frac{2 K}{\varepsilon}\right) \psi(n-1)(n-1)^{s+1 / p} .
$$

From (19) and (22) we obtain the estimation (17).

To estimate the norm $\left\|\Psi_{-\beta, n}(\cdot)\right\|_{p^{\prime}}$ for $1<p^{\prime}<\infty$ we use the statement, which was established in [18], and according to which in the case when $\left\{a_{k}\right\}_{k=1}^{\infty}$ is the monotonically nonincreasing sequence of positive numbers such that $\sum_{k=1}^{\infty} a_{k}^{p^{\prime}} k^{p^{\prime}-2}<\infty$, then for arbitrary $n \in \mathbb{N}$ and $\gamma \in \mathbb{R}$ the following estimate holds

$$
\left\|\sum_{k=n}^{\infty} a_{k} \cos (k x+\gamma)\right\|_{p^{\prime}}=O\left(\sum_{k=n}^{\infty} a_{k}^{p^{\prime}} k^{p^{\prime}-2}+a_{n}^{p^{\prime}} n^{p^{\prime}-1}\right)^{1 / p^{\prime}} .
$$


Putting in (23) $a_{k}=\psi(k), \gamma=\frac{\beta \pi}{2}$ we obtain that for $1<p<\infty, \beta \in \mathbb{R}$ and $n \in \mathbb{N}$

$$
\left\|\Psi_{-\beta, n}(\cdot)\right\|_{p^{\prime}}=O\left(\sum_{k=n}^{\infty} \psi^{p^{\prime}}(k) k^{p^{\prime}-2}+\psi^{p^{\prime}}(n) n^{p^{\prime}-1}\right)^{1 / p^{\prime}} .
$$

Then, using [18, Lemma 3], we conclude that for $1<p^{\prime}<\infty, n \in \mathbb{N}$, under condition (6) and imbedding $g_{1 / p} \in \mathfrak{M}_{0}$ the following estimate holds

$$
\psi^{p^{\prime}}(n) n^{p^{\prime}-1}=O\left(\sum_{k=n}^{\infty} \psi^{p^{\prime}}(k) k^{p^{\prime}-2}\right) .
$$

According to the conditions of Theorem 1 we have that $g_{1 / p} \in \mathfrak{M}_{0}$, so taking into account (25), from (24), we obtain

$$
\left\|\Psi_{-\beta, n}(\cdot)\right\|_{p^{\prime}}=O\left(\sum_{k=n}^{\infty} \psi^{p^{\prime}}(k) k^{p^{\prime}-2}\right)^{1 / p^{\prime}}, \quad 1<p^{\prime}<\infty, \quad \beta \in \mathbb{R}, \quad n \in \mathbb{N} .
$$

Combining (16), (17) and (26) in the case when $g_{1 / p} \in \mathfrak{M}_{0}$, and $g_{s+1 / p} \in G M^{+} \cap G A^{+}$, we arrive at the estimate

$$
\mathcal{E}\left(C_{\beta, p^{\prime}}^{\psi} ; Z_{n-1}^{s}\right)_{C}=O\left(\sum_{k=n}^{\infty} \psi^{p^{\prime}}(k) k^{p^{\prime}-2}\right)^{1 / p^{\prime}}, \quad 1<p<\infty, \quad \frac{1}{p}+\frac{1}{p^{\prime}}=1 .
$$

As follows from [18, Corollary 1 and 2], for $1<p<\infty, 1 / p+1 / p^{\prime}=1, n \in \mathbb{N}$ and $\beta \in \mathbb{R}$, under conditions (6) and (10) and imbedding $g_{1 / p} \in \mathfrak{M}_{0}$ for $E_{n}\left(C_{\beta, p}^{\psi}\right)_{C}$ we arrive at the following order estimates

$$
E_{n}\left(C_{\beta, p}^{\psi}\right)_{C} \asymp\left(\sum_{k=n}^{\infty} \psi^{p^{\prime}}(k) k^{p^{\prime}-2}\right)^{1 / p^{\prime}}
$$

Therefore, by virtue of inequality (4) and relations (27) and (28) we obtain order equality (11).

Further, let us consider the case $p=1$. Let us establish the estimate of the norm $\left\|\Psi_{-\beta, n}(\cdot)\right\|_{p^{\prime}}=\left\|\Psi_{-\beta, n}(\cdot)\right\|_{\infty}$. It is obvious that for any $\beta \in \mathbb{R}$ the following inequality holds

$$
\left\|\Psi_{-\beta, n}(\cdot)\right\|_{\infty}=\left\|\sum_{k=n}^{\infty} \psi(k) \cos \left(k t+\frac{\beta \pi}{2}\right)\right\|_{\infty} \leq \sum_{k=n}^{\infty} \psi(k) .
$$

If $\beta=2 k+1, k \in \mathbb{Z}$, then following estimate takes place

$$
\left\|\Psi_{-\beta, n}(\cdot)\right\|_{\infty}=\left\|\sum_{k=n}^{\infty} \psi(k) \sin k t\right\|_{\infty} \leq(\pi+2) \psi(n) n
$$

(see, e.g. [21, relation (82)]).

According to [21, Lemma 3], if $g_{1} \in \mathfrak{M}_{0}$, where $g_{1}=\{\psi(k) k\}_{k=1}^{\infty}$ and the condition (7) holds, then the following estimates are true

$$
\psi(n) n=O\left(\sum_{k=n}^{\infty} \psi(k)\right)
$$


If $g_{1} \in \mathfrak{M}_{0}$ and the conditions (7) hold, then combining (16), (17), (29) - (31), we obtain the following estimates

$$
\mathcal{E}\left(C_{\beta, 1}^{\psi} ; Z_{n-1}^{s}\right)_{C}= \begin{cases}O\left(\sum_{k=n}^{\infty} \psi(k)\right), & \cos \frac{\beta \pi}{2} \neq 0 \\ O(\psi(n) n), & \cos \frac{\beta \pi}{2}=0 .\end{cases}
$$

To estimate the quantity $\mathcal{E}\left(C_{\beta, 1}^{\psi} ; Z_{n-1}^{s}\right)_{C}$ from below, we use [21, Theorems 3 and 4], according to which, if $g_{1} \in \mathfrak{M}_{0}$ and the conditions (7) and (12) are true, then for $n \in \mathbb{N}$ and $\beta \in \mathbb{R}$ the following the order equalities take place

$$
E_{n}\left(C_{\beta, 1}^{\psi}\right)_{C} \asymp \begin{cases}\sum_{k=n}^{\infty} \psi(k), & \cos \frac{\beta \pi}{2} \neq 0, \\ \psi(n) n, & \cos \frac{\beta \pi}{2}=0 .\end{cases}
$$

The estimate (13) follows from the inequality (4), estimates (32) and (33).

Assume that the conditions of Theorem 1 take place, moreover, more stronger imbedding $g_{1 / p} \in \mathfrak{M}_{C}$ holds. As it follows from [18, Lemma 3], if $g_{1 / p} \in \mathfrak{M}_{C}$ and the condition (6) holds, then for $1<p<\infty$ the following estimates take place

$$
\sum_{k=n}^{\infty} \psi^{p^{\prime}}(k) k^{p^{\prime}-2} \asymp \psi^{p^{\prime}}(n) n^{p^{\prime}-1}
$$

In addition, as it was shown in [21, Lemma 3], if $g_{1} \in \mathfrak{M}_{C}$ and the condition (7) holds, then the following order estimates are true

$$
\sum_{k=n}^{\infty} \psi(k) \asymp \psi(n) n
$$

Formulas (34) and (35), and Theorem 1 allow us to write the following statement.

Theorem 2. Let $s>0,1 \leq p<\infty, g_{1 / p} \in \mathfrak{M}_{C}, g_{s+1 / p} \in G M^{+} \cap G A^{+}, \beta \in \mathbb{R}$ and $n \in \mathbb{N}$.

In the case $1<p<\infty$, if the conditions (6) and (10) hold, then the following order estimates take place

$$
E_{n}\left(C_{\beta, p}^{\psi}\right)_{C} \asymp \mathcal{E}\left(C_{\beta, p^{\prime}}^{\psi} ; Z_{n-1}^{s}\right)_{C} \asymp \psi(n) n^{1 / p},
$$

and in the case $p=1$ if the conditions (7) and (12) hold, then the following order estimates take place

$$
E_{n}\left(C_{\beta, 1}^{\psi}\right)_{C} \asymp \mathcal{E}\left(C_{\beta, 1}^{\psi} ; Z_{n-1}^{s}\right)_{C} \asymp \psi(n) n .
$$

Proof. Order estimates (36) were established in [14]. Note, that when $1<p<\infty, g_{1 / p} \in \mathfrak{M}_{0}$ and

$$
\lim _{t \rightarrow \infty} \alpha\left(g_{1 / p} ; t\right)=\infty,
$$

then the order estimates (36) do not take place, since in this case we have the following (see [18])

$$
\psi(n) n^{\frac{1}{p}}=o\left(\left(\sum_{k=n}^{\infty} \psi^{p^{\prime}}(k) k^{p^{\prime}-2}\right)^{1 / p^{\prime}}\right), \quad n \rightarrow \infty
$$


Similarly, when $p=1, g_{1 / p}=g_{1} \in \mathfrak{M}_{0}$ and

$$
\lim _{t \rightarrow \infty} \alpha\left(g_{1} ; t\right)=\infty
$$

then as follows from [21, Lemma 3]

$$
\psi(n) n=o\left(\sum_{k=n}^{\infty} \psi(k)\right)
$$

in this case, for $\beta$ such that $\cos \frac{\beta \pi}{2} \neq 0$ order estimates (37) do not take place.

As example of the function $\psi(t)$, for which the conditions of Theorem 1 and the equalities (38) and (39) take place, we can use the function

$$
\psi(t)=t^{-1 / p} \ln ^{-\gamma}(t+K), \quad \gamma>\left\{\begin{array}{ll}
\frac{1}{p^{\prime},} & 1<p<\infty, \\
1, & p=1,
\end{array} \quad K> \begin{cases}e^{\gamma p^{\prime} / 2}, & 1<p<\infty, \\
e^{\gamma}, & p=1,\end{cases}\right.
$$

(see $[18,21])$. Let us write the order estimates for the quantities $E_{n}\left(C_{\beta, p}^{\psi}\right)_{C}$ and $\mathcal{E}\left(C_{\beta, p}^{\psi} ; Z_{n-1}^{s}\right)_{C}$ in the case, when $\psi(t)$ has the form (40).

Theorem 3. Let $\psi(t)=t^{-1 / p} \ln ^{-\gamma}(t+K), \beta \in \mathbb{R}$ and $n \in \mathbb{N}$. If $1<p<\infty, \gamma>1 / p^{\prime}$, $K>e^{\gamma p^{\prime} / 2}, 1 / p+1 / p^{\prime}=1$, then

$$
E_{n}\left(C_{\beta, p}^{\psi}\right)_{C} \asymp \mathcal{E}\left(C_{\beta, p^{\prime}}^{\psi} ; Z_{n-1}^{s}\right)_{C} \asymp \psi(n) n^{1 / p} \ln ^{1 / p^{\prime}} n, \quad n \geq 2 ;
$$

if $p=1, \gamma>1, K>e^{\gamma}$, then

$$
E_{n}\left(C_{\beta, 1}^{\psi}\right)_{C} \asymp \mathcal{E}\left(C_{\beta, 1}^{\psi} ; Z_{n-1}^{s}\right)_{C} \asymp\left\{\begin{array}{ll}
\psi(n) n \ln n, & \cos \frac{\beta \pi}{2} \neq 0, \\
\psi(n) n, & \cos \frac{\beta \pi}{2}=0,
\end{array} \quad n \geq 2 .\right.
$$

Proof. We show that for the indicated function $\psi$ of the form (40) all conditions of the Theorem 1 are true. Indeed, for $1<p<\infty, \gamma>1 / p^{\prime}, K>e^{\gamma p^{\prime} / 2}$ we have

$$
\sum_{k=n}^{\infty} \psi^{p^{\prime}}(k) k^{p^{\prime}-2}=\sum_{k=n}^{\infty} \frac{1}{k \ln ^{\gamma p^{\prime}}(k+K)}<\infty, \quad \alpha\left(g_{1 / p} ; t\right)=\frac{(t+K) \ln (t+K)}{\gamma t}>\frac{\ln \left(t+e^{\gamma p^{\prime} / 2}\right)}{\gamma},
$$

and hence $\lim _{t \rightarrow \infty} \alpha\left(g_{1 / p} ; t\right)=\infty$ and $\alpha\left(g_{1 / p} ; t\right)>\frac{p^{\prime}}{2}$.

For $p=1, \gamma>1, K \geq e^{\gamma}$, we have

$$
\sum_{k=n}^{\infty} \psi(k) \leq \sum_{k=n}^{\infty} \frac{1}{k \ln ^{\gamma}\left(k+e^{\gamma}\right)}<\infty, \quad \alpha\left(g_{1} ; t\right)>\frac{\ln \left(t+e^{\gamma}\right)}{\gamma}
$$

and hence $\lim _{t \rightarrow \infty} \alpha\left(g_{1} ; t\right)=\infty$ and $\alpha\left(g_{1} ; t\right)>1$.

It is obvious that for any $s>0$ and $1 \leq p<\infty$ the functions $g_{s+1 / p}(t)=t^{s} \ln ^{-\gamma}(t+K)$ increase monotonically, starting from some point $t_{0}$. Therefore, it is not difficult to be convinced that the sequence $g_{s+1 / p}(k)$ belongs to the set $G M^{+} \cap G A^{+}$.

Therefore, the function $\psi$ of the form (40) satisfies the conditions of Theorem 1. 
Further, using [18, formula (79)], we obtain

$$
\begin{aligned}
\left(\sum_{k=n}^{\infty} \psi^{p^{\prime}}(k) k^{p^{\prime}-2}\right)^{1 / p^{\prime}} & \asymp\left(\int_{n}^{\infty} \psi^{p^{\prime}}(t) t^{p^{\prime}-2} d t\right)^{1 / p^{\prime}}=\left(\int_{n}^{\infty} \frac{d t}{t \ln ^{\gamma p^{\prime}}(t+K)}\right)^{1 / p^{\prime}} \asymp \ln ^{1 / p^{\prime}-\gamma} n \\
& =\psi(n) n^{1 / p} \ln ^{1 / p^{\prime}} n \frac{\ln ^{-\gamma} n}{\ln ^{-\gamma}(n+K)} \asymp \psi(n) n^{1 / p} \ln ^{1 / p^{\prime}} n, \quad n \geq 2 .
\end{aligned}
$$

Then formula (41) follows from the estimate (11) and the above relations.

Similarly, by virtue of [21, inequality (87)] we get

$$
\sum_{k=n}^{\infty} \psi(k) \asymp \int_{n}^{\infty} \psi(t) d t=\int_{n}^{\infty} \frac{d t}{t \ln ^{\gamma}(t+K)} \asymp \ln ^{1-\gamma} n \asymp \psi(n) n \ln n, \quad n>2 .
$$

Formula (42) follows from the estimates (13) and relations (43), in the case where $\beta$ is such that $\cos \frac{\beta \pi}{2} \neq 0$.

As it was already mentioned, for $s=1$ the Zygmund sums $Z_{n-1}^{s}$ coincide with the known Fejér sums $\sigma_{n-1}$. Therefore, Theorem 1 and 2 imply the following statements.

Proposition 1. Let $1 \leq p<\infty, g_{1 / p} \in \mathfrak{M}_{0}, g_{1+1 / p} \in G M^{+} \cap G A^{+}, \beta \in \mathbb{R}$ and $n \in \mathbb{N}$.

In the case $1<p<\infty$, if the conditions (6) and (10) hold, then the following order estimates take place

$$
E_{n}\left(C_{\beta, p}^{\psi}\right)_{C} \asymp \mathcal{E}\left(C_{\beta, p}^{\psi} ; \sigma_{n-1}\right)_{C} \asymp\left(\sum_{k=n}^{\infty} \psi^{p^{\prime}}(k) k^{p^{\prime}-2}\right)^{1 / p^{\prime}} ;
$$

in the case $p=1$, if the conditions (7) and (12) hold, then the following order equlaities take place

$$
E_{n}\left(C_{\beta, 1}^{\psi}\right)_{C} \asymp \mathcal{E}\left(C_{\beta, 1}^{\psi} ; \sigma_{n-1}\right)_{C} \asymp \begin{cases}\sum_{k=n}^{\infty} \psi(k), & \cos \frac{\beta \pi}{2} \neq 0, \\ \psi(n) n, & \cos \frac{\beta \pi}{2}=0 .\end{cases}
$$

Proposition 2. Let $1 \leq p<\infty, g_{1 / p} \in \mathfrak{M}_{C}, g_{1+1 / p} \in G M^{+} \cap G A^{+}, \beta \in \mathbb{R}$ and $n \in \mathbb{N}$.

In the case $1<p<\infty$, if the conditions (6) and (10) hold, then the following order estimates take place

$$
E_{n}\left(C_{\beta, p}^{\psi}\right)_{C} \asymp \mathcal{E}\left(C_{\beta, p}^{\psi} ; \sigma_{n-1}\right)_{C} \asymp \psi(n) n^{1 / p} ;
$$

in the case $p=1$, if the conditions (7) and (12) hold, then the following order estimates take place

$$
E_{n}\left(C_{\beta, 1}^{\psi}\right)_{C} \asymp \mathcal{E}\left(C_{\beta, 1}^{\psi} ; \sigma_{n-1}\right)_{C} \asymp \psi(n) n
$$

\section{References}

[1] Belov A.S. Remarks on Mean Convergence (Boundedness) of Partial Sums of Trigonometric Series. Math. Notes 2002, 71 (5-6), 739-748. doi:10.1023/A:1015860510199 (translation of Mat. Zametki 2002, 71 (6) 807-817. doi:10.4213/mzm386 (in Russian))

[2] Bushev D.M. Approximation of classes of continuous periodic functions by Zygmund sums. Preprint. Inst. Math. AN USSR, Kyiv, 1984. (in Russian)

[3] Dzyadyk V.K. On best approximation in classes of periodic functions defined by integrals of a linear combination of absolutely monotonic kernels. Math. Notes 1974, 16 (5), 1008-1014. doi:10.1007/BF01149788 (translation of Mat. Zametki 1974, 16 (5), 691-701. (in Russian)) 
[4] Hrabova U.Z., Serdyuk A.S. Order estimates for the best approximations and approximations by Fourier sums of the classes of $(\psi, \beta)$-differential functions. Ukrainian Math. J. 2014, 65 (9), 1319-1331. doi:10.1007/s11253-014-0861-7 (translation of Ukrain. Mat. Zh. 2013, 65 (9), 1186-1197. (in Ukrainian))

[5] Kamzolov A.I. Approximation of the functional classes $\widetilde{W}_{p}^{\alpha}(L)$ in the spaces $\mathcal{L}_{p}[-\pi, \pi]$ by the Fejér method. Math. Notes 1978, 23 (3), 185-189. doi:10.1007/BF01651429 (translation of Mat. Zametki 1978, 23 (3), 343-349. (in Russian))

[6] Kostich M. V. Approximation of functions from Weyl-Nagy classes by Zygmund averages. Ukrainian Math. J. 1998, 50 (5), 834-838. doi:10.1007/BF02514336 (translation of Ukrain. Mat. Zh. 199850 (5), 735-738. (in Ukrainian))

[7] Nagy B.Sz. Sur une classe générale de procèdès de sommation pour les sèries de Fourier. Acta Math. Hungar. 1948, $1(3), 14-52$.

[8] Nikol'skii S.M. Approximation of periodic functions by trigonometric polynomials. Tr. Mat. Inst. Steklova 1945, 15, 3-76. (in Russian)

[9] Pinkus A. $n$-Widths in approximation theory. Springer-Verlag, Berlin, 1985. doi:10.1007/978-3-642-69894-1

[10] Serdyuk A.S. On the best approximation of classes of convolutions of periodic functions by trigonometric polynomials. Ukrainian Math. J. 1995, 47 (9), 1435-1440. doi:10.1007/BF01057518 (translation of Ukrain. Mat. Zh. 1995, 47 (9), 1261-1265. (in Russian))

[11] Serdyuk A.S. Widths and best approximations for classes of convolutions of periodic functions. Ukrainian Math. J. 1999, 51 (5), 748-763. doi:10.1007/BF02591709 (translation of Ukrain. Mat. Zh. 1999, 51 (5), 674-687. (in Ukrainian))

[12] Serdyuk A.S. On best approximation in classes of convolutions of periodic functions. Approx. Theory of Functions and Related Problems. Proc. Inst. Math. NAS Ukr. 2002, 35, 172-194. (in Ukrainian)

[13] Serdyuk A.S. Best approximations and widths of classes of convolutions of periodic functions of high smoothness. Ukrainian Math. J. 2005, 57 (7), 1120-1148. doi:10.1007/s11253-005-0251-2 (translation of Ukrain. Mat. Zh. 2005, 57 (7), 946-971. (in Ukrainian))

[14] Serdyuk A.S., Hrabova U.Z. Estimates of uniform approximations by Zygmund sums on classes of convolutions of periodic functions. Approx. Theory of Functions and Related Problems. Proc. Inst. Math. NAS Ukr. 2013, 10 (1), 222-244. (in Ukrainian)

[15] Serdyuk A. S., Sokolenko I. V. Uniform approximation of the classes of $(\psi, \beta)$-differentiable functions by linear methods. Extremal Problems of the Theory of Functions and Related Problems. Proc. Inst. Math. NAS Ukr. 2011, 8 (1), 181-189. (in Ukrainian)

[16] Serdyuk A.S., Sokolenko I. V. Asymptotic estimates for the best uniform approximations of classes of convolution of periodic functions of high smoothness. Ukr. Mat. Visn. 2020, 17 (3), 396-413. (in Ukrainian)

[17] Serdyuk A.S., Stepanyuk T.A. Estimations of the best approximations for the classes of infinitely differentiable functions in uniform and integral metrics. Ukrainian Math. J. 2014, 66 (9), 1393-1407. doi:10.1007/s11253-015-1018-Z (translation of Ukrain. Mat. Zh. 2014, 66 (9), 1244-1256. (in Ukrainian))

[18] Serdyuk A.S., Stepanyuk T.A. Order estimates for the best approximations and approximations by Fourier sums in the classes of convolutions of periodic functions of low smoothness in the uniform metric. Ukrainian Math. J. 2014, 66 (12), 1862-1882. doi:10.1007/s11253-015-1056-6 (translation of Ukrain. Mat. Zh. 2014, 66 (12), 1658-1675. (in Ukrainian))

[19] Serdyuk A.S., Stepanyuk T.A. Uniform approximations by Fourier sums in classes of generalized Poisson integrals. Anal. Math. 2019, 45 (1), 201-236. doi:10.1007/s10476-018-0310-1

[20] Stepanets A.I. Methods of Approximation Theory. Walter de Gruyter, Berlin, 2005.

[21] Stepanyuk T.A. Estimates for the best approximations and approximation by Fourier sums of classes of convolutions of periodic functions of not high smoothness in integral metrics. Approx. Theory of Functions and Related Problems. Proc. Inst. Math. NAS Ukr. 2014, 11 (3), 241-269. (in Ukrainian)

[22] Sz.-Nagy B. Über gewisse Extremalfragen bei transformierten trigonometrischen Entwicklungen. Berichte der math.-phys. Kl. Akad. der Wiss. zu Leipzig, 1938, 90, 103-134. 
[23] Telyakovskii S. A. On the norms of trigonometric polynomials and approximation of differentiable functions by linear averages of their Fourier series. Tr. Mat. Inst. Steklova 1961, 62, 61-97. (in Russian)

[24] Temlyakov V.N. Approximation of periodic functions. Computational Mathematics and Analysis Series. Nova Science Publ. Inc., NY, 1993.

[25] Tikhomirov V.M. Some questions in approximation theory. Moscow Univ. Publ., Moscow, 1976. (in Russian)

[26] Tikhonov S. Trigonometric series with general monotone coefficients. J. Math. Anal. Appl. 2007, 326 (1), $721-735$. doi:10.1016/j.jmaa.2006.02.053

[27] Zygmund A. Smooth functions. Duke Math. J. 1945, 12 (1), 47-76. doi:10.1215/S0012-7094-45-01206-3

[28] Zygmund A. Trigonometric series. Vol. 2. Mir, Moscow, 1965. (in Russian)

Received 01.11.2020

Сердюк А.С., Грабова У.З. Порядкові оиінки рівномірних наближень сумами Зигмунда на класах згорток періодичних функиій // Карпатські матем. публ. - 2021. — Т.13, №1. - С. 68-80.

Суми Зигмунда $Z_{n-1}^{s}(f ; t)$ функції $f \in L_{1}$ - це тригонометричні поліноми вигляду $Z_{n-1}^{s}(f ; t):=\frac{a_{0}}{2}+\sum_{k=1}^{n-1}\left(1-\left(\frac{k}{n}\right)^{s}\right)\left(a_{k}(f) \cos k t+b_{k}(f) \sin k t\right), s>0$, де $a_{k}(f)$ i $b_{k}(f)$ - коефiцієнти Фур'є функції $f$. Отримано точні порядкові оцінки рівномірних наближень сумами Зиг мунда $Z_{n-1}^{s}$ на класах $C_{\beta, p}^{\psi}$. Ці класи складаються з $2 \pi$-періодичних неперервних функцій $f$, які зображаються у вигляді згортки функцій, що належать одиничним кулям просторів $L_{p}$, $1 \leq p<\infty$, з фіксованими твірними ядрами $\Psi_{\beta}(t) \sim \sum_{k=1}^{\infty} \psi(k) \cos \left(k t+\frac{\beta \pi}{2}\right), \Psi_{\beta} \in L_{p^{\prime}}, \beta \in \mathbb{R}$, $\frac{1}{p}+\frac{1}{p^{\prime}}=1$, у випадку, коли добуток $\psi(k) k^{s+1 / p}$ узагальнено монотонно зростає з деякою степеневою швидкістю, i, крім того, при $1<p<\infty$ виконується нерівність $\sum_{k=n}^{\infty} \psi^{p^{\prime}}(k) k^{p^{\prime}-2}<\infty$, а при $p=1-$ нерівність $\sum_{k=n}^{\infty} \psi(k)<\infty$. Показано, що при виконанні зазначених умов суми Зигмунда $Z_{n-1}^{s}$, а також суми Фейєра $\sigma_{n-1}=Z_{n-1}^{1}$ реалізують порядки найкращих рівномірних наближень тригонометричними поліномами на вказаних функціональних класах, а саме при $1<p<\infty$

$$
E_{n}\left(C_{\beta, p}^{\psi}\right)_{C} \asymp \mathcal{E}\left(C_{\beta, p^{\prime}}^{\psi} ; Z_{n-1}^{s}\right)_{C} \asymp\left(\sum_{k=n}^{\infty} \psi^{p^{\prime}}(k) k^{p^{\prime}-2}\right)^{1 / p^{\prime}}, \quad \frac{1}{p}+\frac{1}{p^{\prime}}=1,
$$

а при $p=1$

дe

$$
\begin{array}{ll}
E_{n}\left(C_{\beta, 1}^{\psi}\right)_{C} \asymp \mathcal{E}\left(C_{\beta, 1}^{\psi} ; Z_{n-1}^{s}\right)_{C} \asymp \sum_{k=n}^{\infty} \psi(k), & \cos \frac{\beta \pi}{2} \neq 0, \\
E_{n}\left(C_{\beta, p}^{\psi}\right)_{C} \asymp \mathcal{E}\left(C_{\beta, p^{\prime}}^{\psi} ; Z_{n-1}^{s}\right)_{C} \asymp \psi(n) n, \quad & \cos \frac{\beta \pi}{2}=0,
\end{array}
$$

$$
E_{n}\left(C_{\beta, p}^{\psi}\right)_{C}:=\sup _{f \in C_{\beta, p}^{\psi}} \inf _{t_{n-1} \in \mathcal{T}_{2 n-1}}\left\|f(\cdot)-t_{n-1}(\cdot)\right\|_{C}
$$

$\mathcal{T}_{2 n-1}-$ підпростір тригонометричних поліномів $t_{n-1}$ порядку $n-1$ з дійсними коефіцієнтами,

$$
\mathcal{E}\left(C_{\beta, p}^{\psi} ; Z_{n-1}^{s}\right)_{C}:=\sup _{f \in C_{\beta, p}^{\psi}}\left\|f(\cdot)-Z_{n-1}^{s}(f ; \cdot)\right\|_{C} .
$$

Ключові слова і фрази: найкраще наближення, сума Зигмунда, сума Фейєра, підпростір тригонометричних поліномів, порядкова оцінка. 